\title{
HAUSDORFF MEASURE FUNCTIONS IN THE SPACE OF COMPACT SUBSETS OF THE UNIT INTERVAL BY
}

P. R. GOODEY

\begin{abstract}
The work done in this paper is the result of an attempt to classify those functions $h$ for which the corresponding Hausdorff measure of $\mathscr{F}[0,1]$ is zero. A partial characterization is achieved and in doing this some problems of E. Boardman are solved.
\end{abstract}

Introduction. If $C$ is a compact nonempty subset of $R^{1}$ then $\mathscr{F}[C]$ denotes the compact metric space consisting of all nonempty compact subsets $C$ endowed with the Hausdorff metric. If $h$ is a continuous, increasing function defined on the nonnegative real numbers with $h(0)=0$ we shall denote by $h-m$ the Hausdorff measure generated by $h$ (see [3]). In [1] it is shown that for each function $h$ either $h-m(\mathscr{F}[0,1])=0$ or $\mathscr{F}[0,1]$ has non- $\sigma$-finite $h$ measure. For $\alpha>0$, the functions $h_{\alpha}, g_{\alpha}$ are defined by

$$
h_{\alpha}(t)=2^{-\alpha t^{-1}} \text { and } g_{\alpha}(t)=2^{-t^{-\alpha}} \text { for } t>0
$$

and $h_{\alpha}(0)=g_{\alpha}(0)=0$. Then Boardman shows that $g_{\alpha}-m(\mathscr{F}[0,1])=\infty$ for all $0<\alpha<1$ and that $h_{1}-m(\mathscr{F}[0,1])=0$. The evaluation of

$$
h_{\alpha}-m(\mathscr{F}[0,1]) \text { for } 0<\alpha<1
$$

is left as an open problem.

The work done in this paper is the result of an attempt to classify those functions $h$ for which $h-m(\mathscr{F}[0,1])=0$. The main result is

THEOREM 1. Let $h$ be such that $\liminf _{x \rightarrow 0}\left\{-(x \log h(x))^{-1}\right\}<\infty$, then $h-m(\mathscr{F}[0,1])=0$.

Thus it follows that $h_{\alpha}-m(\mathscr{F}[0,1])=0$ whenever $0<\alpha \leqslant 1$. We observe, from [1], that if there is an $\alpha<1$ with $\liminf _{x \rightarrow 0}\left\{-\left(x^{\alpha} \log h(x)\right)^{-1}\right\}>0$, then $h-m(\mathscr{F}[0,1])=\infty$. It is clear, therefore, that this characterization is only

Received by the editors January 29, 1974 and, in revised form, November 25, 1975. AMS (MOS) subject classifications (1970). Primary 28A10. 
partial as it gives no information about those functions for which $\lim _{x \rightarrow 0}\left\{-(x \log h(x))^{-1}\right\}=\infty$, and yet $\liminf _{x \rightarrow 0}\left\{-\left(x^{\alpha} \log h(x)\right)^{-1}\right\}=0$ for all $\alpha<1$. We shall denote by $\mathcal{X}$ the collection of all such functions. Now if $f_{n}(x)=2^{-(n x)^{-1}}$, then, by Theorem $1, f_{n}-m(\mathscr{F}[0,1])=0$ for $n=1,2, \ldots$ But by a result of Rogers and Taylor [4] there is a function $h$ such that $h-m(\mathcal{F}[0,1])=0$ and $\lim _{x \rightarrow 0+} f_{n}(x)(h(x))^{-1}=0$ for $n=1,2, \ldots$. So there is a function $h \in \mathcal{X}$ for which $h-m(\mathcal{F}[0,1])=0$. Conversely, it follows, by a slight generalization of the methods of [1], that

THEOREM 2. There is a function $h \in \mathcal{X}$ for which $h-m(\mathscr{F}[0,1])=\infty$.

If we combine these results with some work of Dvoretzky [2] we see that if the unit interval has $\sigma$-finite $h$-measure then $2^{-h^{-1}}-m(\mathscr{F}[0,1])=0$. This is interesting in that it relates some properties of $[0,1]$ to some of $\mathscr{F}[0,1]$, and thus might suggest working in the more general setting of an arbitrary compact set $K$ in place of $[0,1]$. Unfortunately, one of our examples, combined with Dvoretzky's result also shows there is a function $h$ such that the unit interval has non-o-finite $h$-measure and yet $2^{-h^{-1}}-m(\mathscr{F}[0,1])=0$.

I should like to thank E. Boardman for providing me with a preprint of her paper [1] and for the interesting correspondence we had concerning this work. I am also indebted to the referee for his constructive criticism of an earlier form of this paper.

Prellminaries. Let $C$ be a compact nonempty subset of $R^{1}$ and let $x_{i} \in C$ for $i=1,2, \ldots, n$; then $\left\{x_{1}, x_{2}, \ldots, x_{n}\right\} \in \mathscr{G}[C]$. The sphere in $\mathscr{F}[C]$, centre $\left\{x_{1}, x_{2}, \ldots, x_{n}\right\}$ and radius $r$ is precisely the set of compact subsets $K$ of $C$ such that $K \cap\left[x_{i}-r, x_{i}+r\right] \neq \varnothing$ for $i=1,2, \ldots, n$. Also if $C \subset \cup_{i=1}^{t} I_{i}$ where the $I_{i}$ are closed intervals each of length $l$, then for $K \in \mathscr{F}[C], K \in \mathscr{F}\left[\cup_{i \in A} I_{i}\right]$ and $K \cap I_{i} \neq \varnothing$ for each $i \in A$ where $A$ is some nonempty subset of $\{1,2, \ldots, t\}$. In fact $K$ belongs to the sphere in $\mathscr{F}\left[\cup_{i=1}^{t} I_{i}\right]$, centre $\left\{x_{i}\right\}_{i \in A}$ and radius $\frac{1}{2} l$ where $x_{i}$ is the midpoint of $I_{i}$. Thus $\mathscr{F}[C]$ is contained in a union of $2^{t}-1$ spheres each of diameter $l$.

\section{Prool of Theorem 1.}

LEMMA 1. Let $\alpha \in(0,1), S=\left\{x_{i}\right\}$ be a sequence of positive real numbers with $\lim _{i \rightarrow \infty} x_{i}=0$ and $I_{j}(j=1,2, \ldots, t)$ be closed intervals such that $\sum_{j=1}^{t} d\left(I_{j}\right)$ $<\alpha$. Then, given any $\varepsilon>0$, there is a sequence $\left\{g_{n}\right\}_{n=1}^{N}$ of sets such that $\mathscr{F}\left[\cup_{j=1}^{t} I_{j}\right] \subset \cup_{n=1}^{N} \mathcal{G}_{n}, \sum_{n=1}^{N} h_{\alpha}\left(d\left(\mathcal{G}_{n}\right)\right)<\varepsilon$ and $d\left(\mathcal{G}_{n}\right) \in S$ for $n=1,2, \ldots$, $N$.

Proor. Let $\eta>0$ be such that $\sum_{j=1}^{t} d\left(I_{j}\right)<\alpha-\eta$. Choose $i_{0}$ such that, for $i \geqslant i_{0}, 2^{t-\eta x_{i}^{-1}}<\varepsilon$. Now $I_{j}$ can be covered by $\left[d\left(I_{j}\right) x_{i}^{-1}\right]+1$ closed intervals each length $x_{i}$, where the square brackets denote the integer part 
function. Thus $\cup_{j=1}^{t} I_{j}$ can be covered by $N(i)=\sum_{j=1}^{t}\left\{\left[d\left(I_{j}\right) x_{i}^{-1}\right]+1\right\}$ closed intervals each of length $x_{i}$. Now

$$
N(i) \leqslant x_{i}^{-1} \sum_{j=1}^{t} d\left(I_{j}\right)+t<(\alpha-\eta) x_{i}^{-1}+t
$$

and so

$$
\left(2^{N(i)}-1\right) h_{\alpha}\left(x_{i}\right)<2^{(\alpha-\eta) x_{i}^{-1}+t-\alpha x_{i}^{-1}}<\varepsilon .
$$

Denote by $\left\{\mathcal{G}_{n}\right\}$ any enumeration of the sets of compact subsets of the nonempty subcollections of the covering intervals each of length $x_{i}$. This completes the proof of the lemma.

LeMMA 2. Let $\alpha$ and $S$ be as in Lemma 1 and denote by $\mathscr{F}(\alpha)$ those compact sets of Lebesgue measure $<\alpha$. Then, given any $\varepsilon>0$, there is a sequence $\left\{\Theta_{n}\right\}$ of sets such that $\mathscr{F}(\alpha) \subset \cup_{n=1}^{\infty} \mathcal{G}_{n}, \sum_{n=1}^{\infty} h_{\alpha}\left(d\left(\mathcal{G}_{n}\right)\right)<\varepsilon$ and $d\left(\mathcal{G}_{n}\right) \in S$ for $n=1$, $2, \ldots$

Proor. Let $K \in \mathscr{F}(\alpha)$; then there is a finite covering $J_{1}, J_{2}, \ldots, J_{t}$ of $K$ by open intervals with rational endpoints such that $\sum_{i=1}^{t} d\left(J_{i}\right)<\alpha$. For $i=1$, $2, \ldots, t$ put $I_{i}=J_{i}$. Then the intervals $I_{1}, I_{2}, \ldots, I_{t}$ satisfy the conditions of Lemma 1 and $K \in \mathscr{F}\left[\cup_{j=1}^{t} I_{j}\right]$. Now there are only enumerably many closed intervals with rational endpoints and thus only enumerably many finite unions of such closed intervals. So we may denote by $g_{1}, g_{2}, \ldots$ those of the finite unions of intervals whose total length is less than $\alpha$. Then by Lemma 1 there is, for each integer $j$, a sequence $\left\{\mathcal{G}_{n}^{j}\right\}_{n=1}^{N(j)}$ of sets such that $\mathscr{F}\left[g_{j}\right]$ $\subset \cup_{n=1}^{N(j)} \mathcal{G}_{n}^{j} ; \sum_{n=1}^{N(j)} h_{\alpha}\left(d\left(\mathcal{G}_{j}^{n}\right)\right)<\varepsilon 2^{-j}$ and $d\left(\mathcal{G}_{n}^{j}\right) \stackrel{n=1}{\in} S$ for $n=1,2, \ldots, N(j)$. But we have shown that $\mathscr{F}(\alpha) \subset \cup_{j=1}^{\infty} \mathscr{F}\left[\mathcal{G}_{j}\right]$ and so

$$
\mathscr{F}(\alpha) \subset \bigcup_{j=1}^{\infty} \bigcup_{n=1}^{N(j)} \mathcal{S}_{n}^{j} ; \quad \sum_{j=1}^{\infty} \sum_{j=1}^{N(j)} h_{\alpha}\left(d\left(\mathcal{G}_{n}^{j}\right)\right)<\varepsilon
$$

and $d\left(\mathcal{G}_{n}^{j}\right) \in S$ for $n=1,2, \ldots, N(j)$ and $j=1,2, \ldots$. This completes the proof of the lemma.

For $s=3,4, \ldots$ we put

$$
b_{s}=\left(\frac{2 s-1}{2 s}\right)^{((2 s-1) / 2 s)}\left(\frac{1}{2 s}\right)^{(1 / 2 s)}, \alpha_{s}=\frac{5}{6} \cdot \frac{7}{8} \ldots \frac{2 s-1}{2 s}
$$

and observe, using Stirling's Formula, that $\alpha_{s}=O\left(s^{-1 / 2}\right)$ as $s \rightarrow \infty$.

Leman 3. Let $S=\left\{x_{i}\right\}$ be as in Lemma 1 and let $I_{1}, I_{2}, \ldots, I_{t}$ be closed intervals such that $\sum_{j=1}^{t} d\left(I_{j}\right) \leqslant 1$. For each $s \geqslant 3$, given any $\varepsilon>0$, there is a 
sequence $\left\{\mathcal{G}_{n}\right\}$ of sets such that $\mathscr{F}\left[\cup_{j=1}^{t} I_{j}\right] \subset \cup_{n=1}^{\infty} \mathcal{G}_{n}, \sum_{n=1}^{a} h_{\alpha_{s}}\left(d\left(\mathcal{G}_{n}\right)\right)<\varepsilon$ and $d\left(\mathcal{G}_{n}\right) \in S$ for $n=1,2, \ldots$

Proor. The proof of this lemma is in two sections.

(a) Let $I_{1}, I_{2}, \ldots, I_{t}$ and $S$ be as in the statement of the lemma. For each $s>3$, given any $\varepsilon>0$, there is a sequence $\left\{\Theta_{n}\right\}$ of sets such that $\mathscr{F}\left[\bigcup_{j=1}^{t} I_{j}\right] \backslash \mathscr{F}((2 s-1) / 2 s) \subset \cup_{n=1}^{\infty} \mathcal{G}_{n}, \sum_{n=1}^{\infty} h_{\alpha_{s}}\left(d\left(\mathcal{G}_{n}\right)\right)<\varepsilon$ and $d\left(\mathcal{G}_{n}\right) \in S$ for $n=1,2, \ldots$.

Now for each $i, \cup_{j=1}^{t} I_{j}$ can be covered by $N(i)=\sum_{j=1}^{t}\left\{\left[d\left(I_{j}\right) x_{i}^{-1}\right]+1\right\}$ closed intervals each of length $x_{i}$. Let $I_{1}^{i}, I_{2}^{i}, \ldots, I_{N(i)}^{i}$ be an enumeration of the intervals. Then if $K \in \mathscr{F}\left[\bigcup_{j=1}^{t} I_{j}\right] \backslash \mathscr{F}((2 s-1) / 2 s)$, cardinality $\left(j: K \cap I_{j}^{i}\right.$ $\neq \varnothing\}>(2 s-1) / 2 s$. Thus, for each $i, \mathscr{F}\left[\cup_{j=1}^{t} I_{j}\right] \backslash \mathscr{F}((2 s-1) / 2 s)$ is contained in a set of $M=\sum\left\{\left(\begin{array}{c}N(i) \\ k\end{array}\right):(2 s-1) / 2 s x_{i} \leqslant k \leqslant N(i)\right\}$ spheres each of diameter $x_{i}$.

Now the number of terms in $M$ is $O\left(x_{i}^{-1}\right)$ and the binomial coefficients can be estimated by Stirling's Formula. We find, for any fixed $\eta>0$, an estimate of the form

$$
M h_{\alpha_{s}}\left(x_{i}\right)=o\left(\left(b_{s}^{-1} 2^{-\alpha_{s}+\eta}\right)^{x_{i}^{-1}}\right) \text { as } i \rightarrow \infty .
$$

So we need to prove that $2^{-\alpha_{s}}<b_{s}$ for all $s \geqslant 3$. This can easily be verified for $s=3$. For $s \geqslant 4$ we use the inequality

$$
(2 s)^{1 / 2}>e>(1+1 /(2 s-1))^{2 s-1}
$$

to show that

$$
\frac{2 s-1}{2 s} \log \frac{2 s-1}{2 s}>\frac{1}{4 s} \log \frac{1}{2 s} .
$$

It is thus sufficient to prove that, for $s \geqslant 4$ we have

$$
\alpha_{s} \log 2+(3 / 4 s) \log (1 / 2 s)>0 .
$$

We observe that since $2 s>e^{2}>(1+1 / s)^{2 s}$ we have

$$
\frac{3}{2(2 s+1)} \log \frac{1}{2 s+2}>\frac{3}{4 s} \log \frac{1}{2 s} \text {. }
$$

Using this fact we can easily deduce the required inequalities by induction on $s$ and so (a) is proved.

(b) Let $S$ be as in the statement of the lemma and let $I_{1}, I_{2}, \ldots, I_{t}$ be closed intervals such that $\sum_{j=1}^{t} d\left(I_{j}\right)<(2 s-1) / 2$ s. For each $s \geqslant 3$, given any $\varepsilon>0$, there is a sequence $\left\{\Theta_{n}\right\}$ of sets such that $\mathscr{F}\left[\bigcup_{j=1}^{t} I_{j}\right] \backslash \mathscr{F}\left(\alpha_{s}\right) \subset \cup_{n=1}^{\infty} \mathcal{G}_{n}$; $\sum_{n=1}^{\infty} h_{\alpha_{s}}\left(d\left(\mathcal{G}_{n}\right)\right)<\varepsilon$ and $d\left(\Theta_{n}\right) \in S$ for $n=1,2, \ldots$ 
This is proved by induction on $s$. For $s=3$ the statement is trivial since in that case $\mathscr{F}\left[\cup_{j=1}^{t} I_{j}\right] \backslash \mathscr{F}\left(\alpha_{s}\right)=\varnothing$. Now assume it is true for some $s \geqslant 3$ and let $I_{1}, I_{2}, \ldots, I_{t}$ be closed intervals with $\sum_{j=1}^{t} d\left(I_{j}\right)<(2 s+1) /(2 s+2)$. Then

$$
\sum_{j=1}^{t} d\left(\frac{2 s+2}{2 s+1} I_{j}\right)<1
$$

and

$$
\begin{aligned}
\mathscr{F}\left[\bigcup_{j=1}^{t} \frac{2 s+2}{2 s+1} I_{j}\right] \backslash \mathscr{F}\left(\alpha_{s}\right) \subset & \left(\mathscr{F}\left[\bigcup_{j=1}^{t} \frac{2 s+2}{2 s+1} I_{j}\right] \mid \mathscr{F}\left(\frac{2 s-1}{2 s}\right)\right) \\
& \cup\left(\mathscr{F}\left(\frac{2 s-1}{2 s}\right) \mid \mathscr{F}\left(\alpha_{s}\right)\right) .
\end{aligned}
$$

But, by (a), there is a sequence $\left\{\mathcal{G}_{n}^{0}\right\}$ of sets such that

$$
\mathscr{F}\left[\bigcup_{j=1}^{t} \frac{2 s+2}{2 s+1} I_{j}\right] \backslash \mathscr{F}\left(\frac{2 s-1}{2 s}\right) \subset \bigcup_{n=1}^{\infty} \mathcal{G}_{n}^{0} ; \quad \sum_{n=1}^{\infty} h_{\alpha_{s}}\left(d\left(\mathcal{G}_{n}^{0}\right)\right)<\frac{1}{2} \varepsilon
$$

and $d\left(\mathcal{G}_{n}^{0}\right) \in(2 s+2) S /(2 s+1)$ for $n=1,2, \ldots$.

Let $g_{1}, g_{2}, \ldots$ be an enumeration of all the finite unions of closed intervals with rational endpoints whose total length is less than $(2 s-1) / 2 s$. Then

$$
\mathcal{F}\left(\frac{2 s-1}{2 s}\right) \backslash \mathcal{F}\left(\alpha_{s}\right) \subset \bigcup_{i=1}^{\infty}\left(\mathcal{F}\left[\mathcal{G}_{i}\right] \backslash \mathcal{F}\left(\alpha_{s}\right)\right) .
$$

By induction hypothesis, for each $i$, there is a sequence $\left\{\mathcal{G}_{n}^{i}\right\}$ of sets such that

$$
\mathscr{F}\left[\mathcal{G}_{i}\right] \backslash \mathcal{F}\left(\alpha_{s}\right) \subset \bigcup_{n=1}^{\infty} \mathcal{S}_{n}^{i} ; \quad \sum_{n=1}^{\infty} h_{\alpha_{s}}\left(d\left(\mathcal{S}_{n}^{i}\right)\right)<\varepsilon 2^{-i-1}
$$

and $d\left(\mathcal{G}_{n}^{i}\right) \in(2 s+2) S /(2 s+1)$ for $n=1,2, \ldots$ Hence

$$
\begin{aligned}
& \mathscr{F}\left[\bigcup_{j=1}^{t} I_{j}\right] \backslash \mathscr{F}\left(\alpha_{s+1}\right) \subset \bigcup_{i=0}^{\infty} \bigcup_{n=1}^{\infty} \frac{2 s+1}{2 s+2} \varsigma_{n}^{i} ; \\
& d\left((2 s+1) \mathcal{G}_{n}^{i} /(2 s+2)\right)=(2 s+1) d\left(\mathcal{G}_{n}^{i}\right) /(2 s+2) \in S \text { for } n=1,2, \ldots \text { and } \\
& i=0,1,2, \ldots ; \text { and }
\end{aligned}
$$

$$
\sum_{i=0}^{\infty} \sum_{n=1}^{\infty} h_{\alpha_{s+1}}\left(\left(d \frac{2 s+1}{2 s+2} \mathcal{G}_{n}^{i}\right)\right)=\sum_{i=0}^{\infty} \sum_{n=1}^{\infty} h_{\alpha_{s}}\left(\left(d \mathcal{G}_{n}^{i}\right)\right)<\varepsilon .
$$

This completes the induction and so (b) is proved.

We saw, in the proof, how (b) implies that given $\varepsilon>0$ there is a sequence $\left\{\mathcal{G}_{n}\right\}$ of sets such that 


$$
\mathscr{F}\left(\frac{2 s-1}{2 s}\right) \backslash \mathscr{F}\left(\alpha_{s}\right) \subset \bigcup_{n=1}^{\infty} \mathcal{G}_{n} ; \quad \sum_{n=1}^{\infty} h_{\alpha_{s}}\left(d\left(\mathcal{G}_{n}\right)\right)<\varepsilon
$$

and $d\left(\mathcal{G}_{n}\right) \in S$ for $n=1,2, \ldots$ Combining this fact with (a) and Lemma 2 we easily deduce Lemma 3.

LEMMA 4. Let $\alpha$ and $S$ be as in Lemma 1 and let $\varepsilon>0$ be given. Then there are sets $\left\{G_{n}\right\}$ such that $\mathscr{F}[0,1] \subset \cup_{n=1}^{\infty} G_{n} ; \sum_{n=1}^{\infty} h_{\alpha}\left(d\left(G_{n}\right)\right)<\varepsilon$ and $d\left(\mathcal{G}_{n}\right) \in S$ for $n=1,2, \ldots$.

Proof. Choose $s \geqslant 3$ so that $0<\alpha_{s}<\alpha$. By Lemma 3 there are sets $\left\{g_{n}\right\}$ such that $\mathscr{F}[0,1] \subset \cup_{n=1}^{\infty} \Theta_{n} ; \sum_{n=1}^{\infty} h_{\alpha_{s}}\left(d\left(\mathcal{G}_{n}\right)\right)<\varepsilon$ and $d\left(\mathcal{G}_{n}\right) \in S$ for $n=1$, $2, \ldots$ Thus

$$
\sum_{n=1}^{\infty} h_{\alpha}\left(d\left(\mathcal{G}_{n}\right)\right)<\sum_{n=1}^{\infty} h_{\alpha_{s}}\left(d\left(\mathcal{G}_{n}\right)\right)<\varepsilon
$$

as required.

Proof of Theorem 1. Let $A=\liminf _{x \rightarrow 0}\left\{-(x \log h(x))^{-1}\right\}$, then $0<A<$ $\infty$. Choose $\alpha \in(0,1)$ so that $A \log 2<\alpha^{-1}$. Then there is a sequence $S=\left\{x_{i}\right\}$ of positive real numbers with $\lim _{i \rightarrow \infty} x_{i}=0$ and $-\left(x_{i} \log h\left(x_{i}\right)\right)^{-1}$ $<(\alpha \log 2)^{-1}$ for $i=1,2, \ldots$. Hence $h\left(x_{i}\right)<h_{\alpha}\left(x_{i}\right)$ for $i=1,2, \ldots$ Thus, by Lemma 4 , given any $\varepsilon>0$ there are sets $\left\{\mathcal{G}_{n}\right\}$ such that $\mathscr{F}[0,1] \subset \cup_{n=1}^{\infty} \mathcal{G}_{n}$; $\sum_{n=1}^{\infty} h_{\alpha}\left(d\left(\mathcal{G}_{n}\right)\right)<\varepsilon$ and $d\left(G_{n}\right) \in S$ for $n=1,2, \ldots$ Hence

$$
\sum_{n=1}^{\infty} h\left(d\left(\mathcal{G}_{n}\right)\right)<\sum_{n=1}^{\infty} h_{\alpha}\left(d\left(\mathcal{G}_{n}\right)\right)<\varepsilon
$$

and so $h-m(\mathscr{F}[0,1])=0$ as required.

\section{REFERENCES}

1. E. Boardman, Some Hausdorf measure properties of the space of compact subsets of $[0,1]$ Quart. J. Math. Oxford Ser. (2) 24 (1973), 333-341. MR 48 \#8728.

2. A. Dvoretzky, A note on Hausdorff dimension functions, Proc. Cambridge Philos. Soc. 44 (1948), 13-16. MR 9, 275.

3. C. A. Rogers, Hausdorff measures, Cambridge Univ. Press, New York and London, 1970. MR 43 \# 7576.

4. C. A. Rogers and S. J. Taylor, Additive set functions in Euclidean space. II, Acta Math. 109 (1963), 207-240. MR 28 \#4070.

Department of Mathematics, Royal Holloway College, University of London, Eghu HILL, EghaM, SURREY TW20 OEX, ENGLAND 